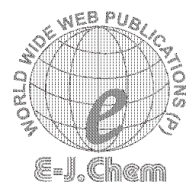

http://www.e-journals.net
ISSN: 0973-4945; CODEN ECJHAO

E-Journal of Chemistry 2009, 6(3), 651-658

\title{
Comparative Molecular Field Analysis (CoMFA) for Thiotetrazole Alkynylacetanilides, a Non-Nucleoside Inhibitor of HIV-1 Double Mutant K103N/Y181C Reverse Transcriptase
}

\author{
SIVAN SREE KANTH, KOTLA SAI ABHISHAKE \\ and MANGA VIJJULATHA* \\ Department of Chemistry, Nizam College, (O.U), \\ Basheerbagh, Hyderabad-500 001, India. \\ sivan.sreekanth@gmail.com,drmvl2002@yahoo.com
}

Received 13 October 2008; Accepted 11 December 2008

\begin{abstract}
Non-nucleoside reverse transcriptase inhibitors (NNRTIs) are allosteric inhibitors of the HIV-1 reverse transcriptase. HIV-1 resistance to nucleoside RT inhibitors such as AZT can arise through mutations in the coding region of RT. Recently a series of thioterazolyl acetanilides were reported as potent inhibitors of HIV-1 wild type and double mutant K103N/Y181C reverse transcriptase. In the present study 3D quantitative structure activity relationship (3D QSAR) studies involving comparative molecular field analysis (CoFMA) were performed on 28 thiotetrazole alkynylacetanilides. CoMFA was performed using sibyl software $6.7 \mathrm{v}$. The molecules were divided into training set and test set. The developed model based on training set containing 20 molecules gave $\mathrm{Q}^{2}$ value of 0.587 and non cross validation $\mathrm{R}^{2}$ value of 0.961 and standard error of estimate 0.098 for CoMFA. The steric and electrostatic contributions are $42.7 \%$ and $57.3 \%$ The cross validation on test set gave $\mathrm{R}^{2}$ value of 0.74 . The CoMFA model provided the most significant correlation of steric and electrostatic fields with biological activities. The information rendered by $3 \mathrm{D}$ QSAR model may afford valuable clues to optimize the lead and design new potential inhibitors
\end{abstract}

Keywords: 3D QSAR, CoMFA, Acquired immuno deficiency syndrome (AIDS), Human infected virus-1 (HIV-1), Non-nucleoside reverse transcriptase inhibitors (NNRTIs). 


\section{Introduction}

Number of people infected with AIDS has increased to an unprecedented level of 39.5 million $^{1}$ throughout the world. Although the introduction of highly active antiretroviral therapy (HAART) in 1996 led to a drastic reduction of AIDS related mortality. Despite the recent availability of simplified regimens, many patients still undergo treatment changes, this is moreover due to treatment failure following appearance of drug resistance ${ }^{2}$ transmission of resistant viruses ${ }^{3}$ are serious concerns and new therapeutic drugs are therefore urgently needed.

HIV-1 RT, which is virally encoded, is essential for the replication of HIV -1 , it converts the viral RNA genome into a double - stranded linear DNA intermediate that is subsequently integrated into the host cell DNA. Two types of drugs that inhibit HIV-1 polymerase activity are nucleoside and non-nucleoside inhibitors. Non-nucleoside reverse transcriptase inhibitors (NNRTIs) are important components of the first line HAART regimens.K103N/Y181C HIV-1 double mutant reverse transcriptase is mutant viral protein were amino acids LYS 103 is mutated by ASN and TYR 181 is mutated by CYS. This mutation leads to broad resistance against all the available non-nucleoside reverse transcriptase inhibitors ${ }^{4}$. Recently thiotetrazole alkynylacetanilides (shown in Figure 1) were reported as potent and bioavailable NNRTIs of the HIV-1 wild type and K103N/Y181C double mutant reverse transcriptases ${ }^{5}$. With these molecules as the base, we initiated a three dimensional QSAR using comparative molecular field analysis (CoMFA). Since drug discovery process is in the new direction involving various computational approaches including 3D QSAR tools like CoMFA, which have been increasingly employed in rational drug discovery process to understand the drug receptor interaction and to design new molecules. A QSAR model establishes a statistical relationship between the biological activity exerted by a series of compounds and a set of parameters determined from their structures. The factors contributing to the biological activity can be understood through use of different physicochemical descriptors in the generation of QSAR models ${ }^{6}$. The mathematical 3D QSAR equations can be computed with the help of a large number of statistical models, such as multilinear regression, partial least squares (PLS), or neural networks. An important component of all QSAR models is a proper validation and evaluation of the prediction power. The present study is aimed to gain insight into the steric and electrostatic properties of these compounds, their influence on the activity and to derive predictive 3D-QSAR models to design new class of inhibitors

\section{Experimental}

For the current 3D QSAR studies of HIV-1 double mutant reverse transcriptase inhibitors, a reported data set of thiotetrazole alkynylacetanilides derivatives were considered to derive $3 \mathrm{D}$ QSAR models. The reported $\mathrm{IC}_{50}$ values were converted into the corresponding $p \mathrm{IC}_{50}$ using the following formula $\mathrm{pIC}_{50}=\log \mathrm{IC}_{50}$ (Table 1$)$. The data set of 28 molecules was grouped into training set and test set containing twenty and eight molecules. The molecules were built, and optimized using Tripos force field, gasteiger-huckel charges, by conjugate gradient method to a convergence gradient of $0.001 \mathrm{Kcals} / \mathrm{mol} / \AA$ before alignment. Structural alignment is the most sensitive and vital part since the interaction energies depend upon the positioning of molecules in $3 \mathrm{D}$ fixed lattice. In the present study the optimized structures were aligned on the template 27 , 
which is the most active molecule among the given set. The aligned molecules are shown in Figure 1<smiles>O=C(CSc1nnnn1-c1cccc2ccccc12)Nc1ccccc1[N+](=O)[O-]</smiles>

Compound 1<smiles>O=C(CSc1nnnn1-c1ccc(Br)cc1Cl)Nc1ccc(P)cc1Cl</smiles>

Compound $\quad R^{1} \quad R^{2}$<smiles>CC(C)(C)c1ccc(-n2nnnc2SCC(=O)Nc2ccccc2Cl)c(Cl)c1</smiles>

Compound 2

$\begin{array}{lllcll}3 & \mathrm{t}-\mathrm{Bu} & \mathrm{CO}_{2} \mathrm{H} & \text { Compound } & \mathrm{R}^{1} & \mathrm{R}^{2} \\ 4 & \mathrm{C}-\mathrm{Pr} & \mathrm{CO}_{2} \mathrm{H} & & & \\ 5 & \mathrm{t}-\mathrm{Bu} & \mathrm{SO}_{2} \mathrm{NH}_{2} & 9 & \mathrm{t}-\mathrm{Bu} & \mathrm{CH}_{2} \mathrm{CH}_{2} \mathrm{COOH} \\ 6 & \mathrm{C}-\mathrm{Pr} & \mathrm{SO}_{2} \mathrm{NH}_{2} & 10 & \mathrm{t}-\mathrm{Bu} & -\mathrm{C} \equiv \mathrm{CCOOH}^{-} \\ 7 & \mathrm{t}-\mathrm{Bu} & \mathrm{SO}_{2} \mathrm{Me} & 11 & \mathrm{t}-\mathrm{Bu} & -\mathrm{C} \equiv \mathrm{CCH}_{2} \mathrm{CH}_{2} \mathrm{OH} \\ 8 & \mathrm{t}-\mathrm{Bu} & 12 & \mathrm{c}-\mathrm{Pr} & -\mathrm{C}_{\equiv} \mathrm{CCH}_{2} \mathrm{CH}_{2} \mathrm{OH}\end{array}$<smiles>CC(C)Cc1ccc(-n2nnnc2SCC(=O)Nc2ccc(C#CP)cc2Cl)c(Cl)c1</smiles>

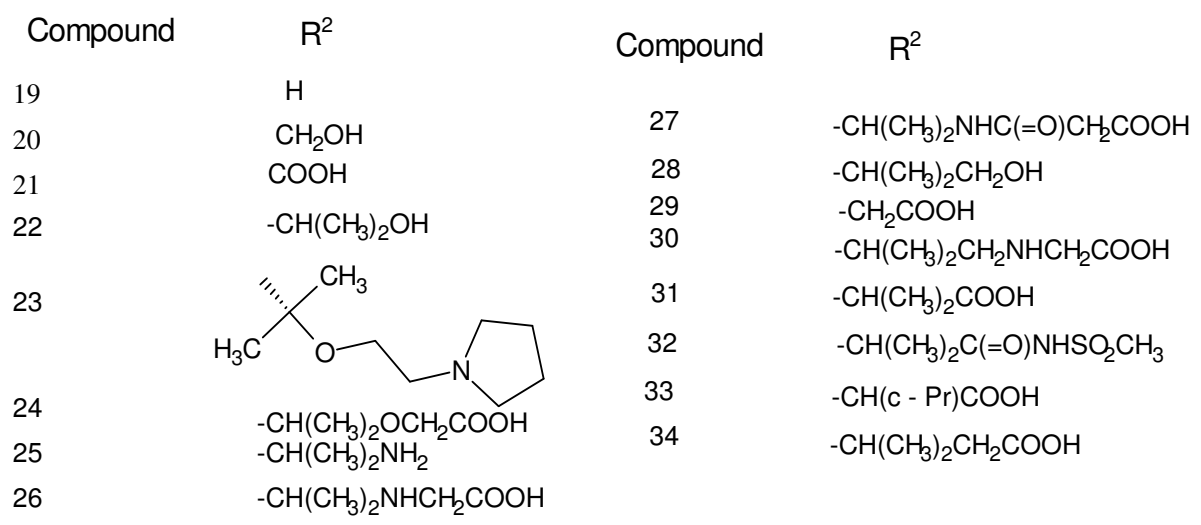

Figure 1. Structure of thiotetrazole alkynylacetanilides. 


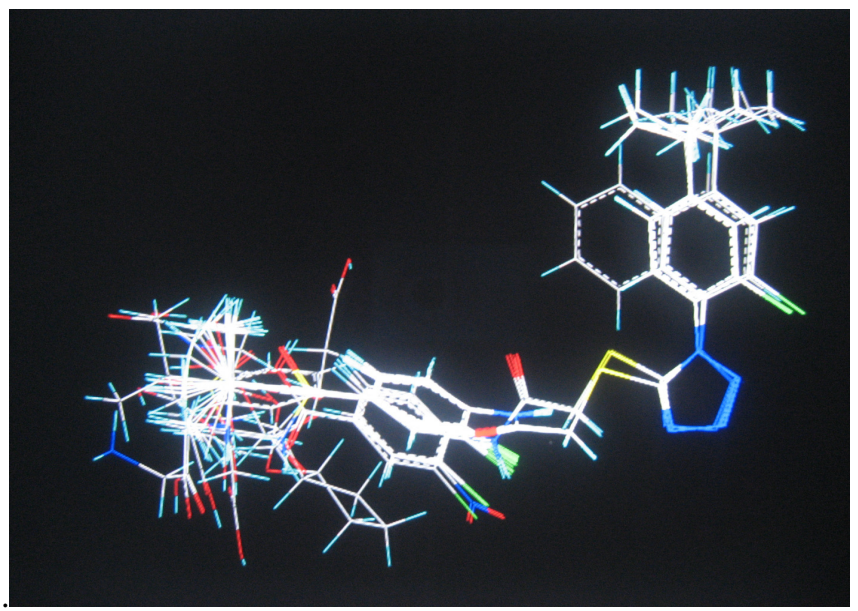

Figure 2. Alignment of all molecules used for CoMFA.

The CoMFA potential fields were calculated at each lattice intersection of a regularly spaced grid of $2.0 \AA$. The grid box dimensions were determined automatically in such a way those region boundaries were extended beyond $4 \AA$ in each direction from coordinates of each molecule. The Vandar Waals potentials and Coulombic terms, which represent steric and electrostatic fields, respectively, were calculated using Tripos force field. A $s p 3$ hybridized carbon atom with radius $1.52 \AA$ bearing +1 charge served as probe atom to calculate steric and electrostatic fields. In CoMFA, Lennard-Jones (6-12 interactions), the steric interaction field and Columbic electrostatic potentials $(1 / r)$ were calculated at each lattice intersection.

Partial Least Square (PLS) analysis implemented in Sybyl was employed to obtain correlation between the descriptors derived by CoMFA and $p \mathrm{IC}_{50}$ values ${ }^{7,8}$. The minimum sigma (column filtering) was set to $2.0 \mathrm{kcal} / \mathrm{mol}$ accelerate the regression analysis and to reduce the noise. Initially, leave one out (LOO) cross validation method was carried out to check the predictivity of the derived model and to determine the optimum number of components with minimum standard error of estimate. The $Q^{2}$ is calculated by the following equation

$$
Q^{2}=1-\Sigma\left(p \mathrm{IC}_{50 \mathrm{obs}}-\mathrm{pIC}_{50 \mathrm{pred}}\right)^{2} / \Sigma\left(\mathrm{pIC}_{50 \mathrm{obs}}-p \mathrm{IC}_{50 \text { mean }}\right)^{2}
$$

The predictive correlation coefficient $\left(\mathrm{R}^{2}\right.$ pred) based on the test molecules, is computed by using formula

$$
\mathrm{R}^{2} \text { pred }=(\mathrm{SD}-\mathrm{PRESS}) / \mathrm{SD}
$$

Where, SD is the sum of the squared deviations between the biological activities of the test set and mean activities of training set molecules and PRESS is the sum of squared deviation between predicted and actual activity for every molecule in test set.

\section{Results and Discussion}

The CoMFA PLS yielded cross-validated $Q^{2}$ value of 0.587 and non-cross validated correlation coefficient $\mathrm{R}^{2}$ of 0.961 . The cross validation performed using test gave a correlation coefficient $\mathrm{R}^{2}$ pred of 0.74 . The steric and electrostatic contributions are $42.7 \%$ and $57.3 \%$. Table 2 lists experimental activities, estimated activities and residual values of the training set and test set by CoMFA model. 
Table 2. Observed and predicted activities of compounds used in training and test set

\begin{tabular}{|c|c|c|c|}
\hline \multicolumn{4}{|c|}{ Training Set } \\
\hline Compound & $p \mathrm{IC}_{50}$ obs & $p \mathrm{IC}_{50}$ pred & residual \\
\hline 1 & 6.12 & 6.078 & 0.042 \\
\hline 3 & 7.18 & 7.162 & 0.018 \\
\hline 4 & 6.82 & 6.847 & -0.027 \\
\hline 6 & 7.35 & 7.377 & -0.027 \\
\hline 7 & 7.58 & 7.51 & 0.07 \\
\hline 8 & 7.78 & 7.778 & 0.002 \\
\hline 9 & 6.82 & 6.834 & -0.014 \\
\hline 10 & 7.42 & 7.532 & -0.112 \\
\hline 11 & 7.92 & 7.974 & -0.054 \\
\hline 19 & 7.36 & 7.383 & -0.023 \\
\hline 20 & 7.7 & 7.547 & 0.153 \\
\hline 22 & 7.77 & 7.634 & 0.136 \\
\hline 24 & 7.64 & 7.582 & 0.058 \\
\hline 25 & 7.42 & 7.617 & -0.197 \\
\hline 27 & 8.1 & 7.945 & 0.155 \\
\hline 28 & 7.77 & 7.748 & 0.022 \\
\hline 29 & 7.5 & 7.602 & -0.102 \\
\hline 30 & 7.64 & 7.654 & -0.014 \\
\hline 32 & 7.72 & 7.714 & 0.006 \\
\hline 34 & 7.58 & 7.671 & -0.091 \\
\hline \multicolumn{4}{|c|}{ Test Set } \\
\hline Compound & $p \mathrm{IC}_{50} \mathrm{obs}$ & $p \mathrm{IC}_{50}$ pred & residual \\
\hline 2 & 7.03 & 6.4 & 0.63 \\
\hline 5 & 7.44 & 7.507 & -0.067 \\
\hline 12 & 8.05 & 7.482 & 0.568 \\
\hline 21 & 7.33 & 7.419 & -0.089 \\
\hline 23 & 7.1 & 7.638 & -0.538 \\
\hline 26 & 7.52 & 7.753 & -0.233 \\
\hline 31 & 7.55 & 7.617 & -0.067 \\
\hline 33 & 7.37 & 7.67 & -0.3 \\
\hline
\end{tabular}

The statistical parameters for the developed CoMFA model are presented in Table 3. These correlation coefficients suggest that our model is reliable and accurate. Figure 2 shows correlation between the experimental and predicted $p \mathrm{IC}_{50}$ values of training and test sets by this CoMFA model. 


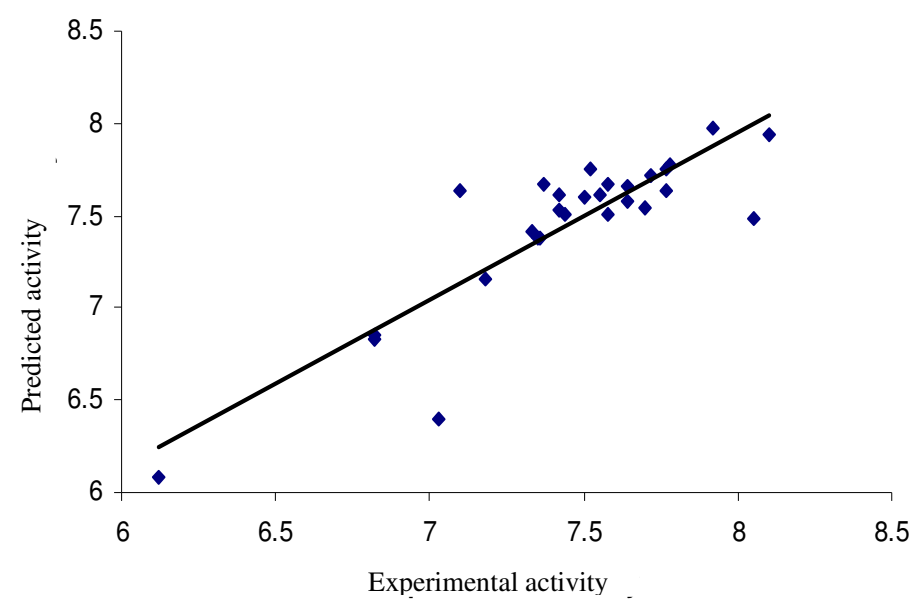

Figure 3. Plot of $p \mathrm{IC}_{50}$ experimental versus $p \mathrm{IC}_{50}$ predicted form $3 \mathrm{D}$ QSAR model.

Table 3. CoMFA PLS results summary.

\begin{tabular}{lc}
\hline \multicolumn{1}{c}{ Index } & CoMFA \\
\hline $\mathrm{Q}^{2}$ LOO & 0.587 \\
$\mathrm{R}^{2}$ & 0.961 \\
$\mathrm{R}^{2}$ pred & 0.74 \\
$\mathrm{n}$ & 3 \\
$F-$ value & 130.108 \\
$\mathrm{SEE}$ & 0.098 \\
Steric & $0.427(42.7 \%)$ \\
Electrostatic & $0.573(57.3 \%)$ \\
PRESS & 0.97 \\
\hline
\end{tabular}

The statistical indices presented in Table 3 are $\mathrm{Q}^{2}$, LOO cross-validated correlation coefficient; $\mathrm{R}^{2}$, non-cross validated correlation coefficient; $\mathrm{R}^{2}$ pred, predictive correlation coefficient; n, number of components used in the PLS analysis; SEE, standard error of estimate; $F$-value, $F$-statistic for analysis.

CoMFA contour maps were generated to visualize the information content of the derived 3D-QSAR model. The contour plots are the representation of the lattice points and the difference in the molecular field values at lattice points is strongly connected with difference in the receptor binding affinity. Molecular fields define the favorable or unfavorable interaction energies of aligned molecules with a probe atom traversing across the lattice grid points surrounding the molecules. The steric interactions are represented by green and yellow colored contours whereas electrostatic interactions are displayed as red and blue contours. Figure 3(a, b) shows the contour maps derived from the CoMFA PLS model. Highly active compound 27 was embedded in the maps to demonstrate its affinity for the steric and electrostatic regions of inhibitors. The $3 \mathrm{D}$ colored plots suggest the modification required to design new molecules. 


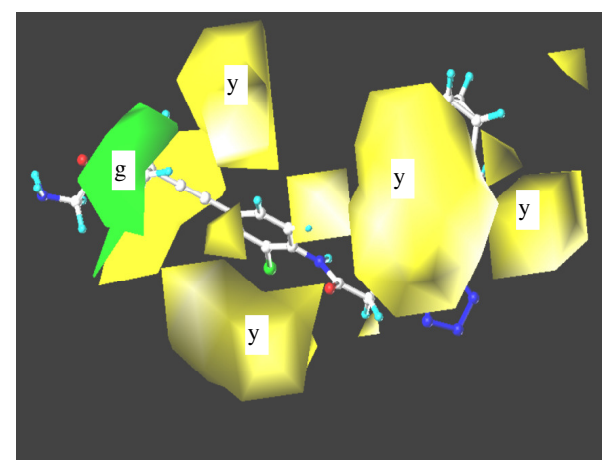

(a)

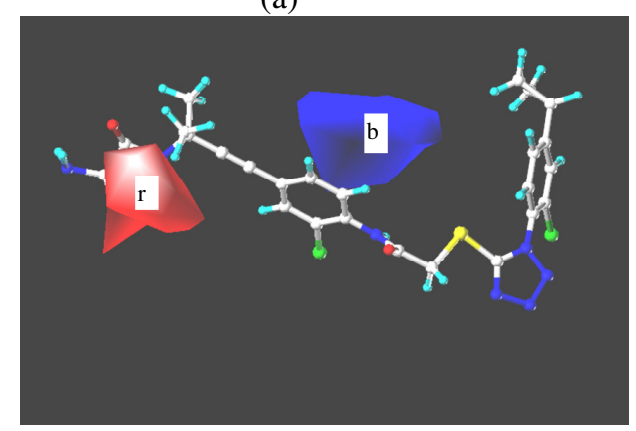

(b)

Figure 4. CoMFA Stdv*Coeff. contour plots (a) the steric field distribution and (b) the electrostatic field distribution for high active compound 27. Green $(\mathrm{g})$ contour maps for sterically favored areas and sterically disfavored areas in yellow (y). Positive potential favored areas in blue (b), negative potential favored areas in red (r).

The countour map of steric fields Figure 4(a) shows a large yellow region near the N1 position of tetrazole ring indicating more bulky group will decrease the activity as shown by compound 1. Substituting the methyl group with more bulky group at alkynyl position will increase the activity as indicated by green region. The countour map of electrostatic fields figure 4(b) show a blue region near the 5,6 position of chlorophenyl acetanilide ring indicate electropositive substituents are favored in the region. The red region near the $\mathrm{NH}$ at the tail region indicates electronegative substituents are favored in the region.

\section{Conclusion}

3D QSAR analysis has been performed on 28 thiotetrazole alkynylacetanilides derivatives as inhibitors of HIV-1 reverse transcriptase double mutant using CoMFA. Stable and statistically reliable predictive model of CoMFA have shown significant $Q^{2}, R^{2}$ and $R^{2}$ pred $(0.587,0.961$ and 0.74 , respectively), indicating a better statistical relationship between the activity and descriptors. Based on this model activities of eight test set molecules have been predicted and this model exhibited good prediction correlation with acceptable error range. The CoMFA results suggest that steric interactions $(42.7 \%)$ as well as electrostatic interactions $(57.3 \%)$ contribute to the activities of inhibitors. The 3D contour plots derived from the CoMFA provided vital clues that can be used to design new molecules with improved inhibitory activity. 


\section{Acknowledgment}

The Authors are thankful to the management of PGRRCDE, Osmania University for providing the software facility. We are also thankful to Dept. of Chemistry, Nizam College for their continuous support.

\section{Reference}

1. AIDS epidemic update, December 2006, UNAIDS/WHO, www.unaids.org.

2. Johnson V A., Brun-Ve'zinet F, Clotet B, Conway B, D’Aquila R T, Demeter L M, Kuritzkes D R, Pillay D, Schapiro J M, Telenti A and Richman D D, Top HIV Med., 2003, 11, 215

3. Turner D and Wainberg M A, AIDS Rev., 2006, 8, 17.

4. Gallant J E, Top HIV Med., 2005, 13, 138.

5. Alexandre G, Ma'an H, Amad, Pierre R Bonneau, Coulombe R, Patrick L, DeRoy, Louise D, Jianmin D, Michel G, Ingrid G, Araz J, Eric J, Serge L, Eric M, Bruno S, and Christiane Y, Bioorg Med Chem Lett., 2007, 17, 4437-4441

6. Vadlamudi S M and Kulkarni V M, Internet Electron J Mol Des., 2004, 3, 586-609.

7. Geladi P, J Chemon., 1998, 2, 231.

8. Cramer R III, Bunce D, Patterson D E and Frank I E, Quant Struct Act Relat., 1988, 7, 18-25. 


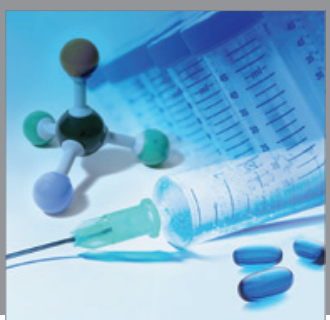

International Journal of

Medicinal Chemistry

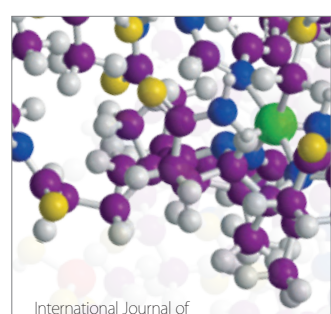

Carbohydrate Chemistry

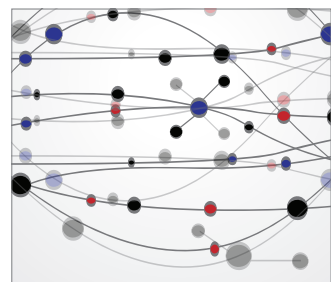

The Scientific World Journal
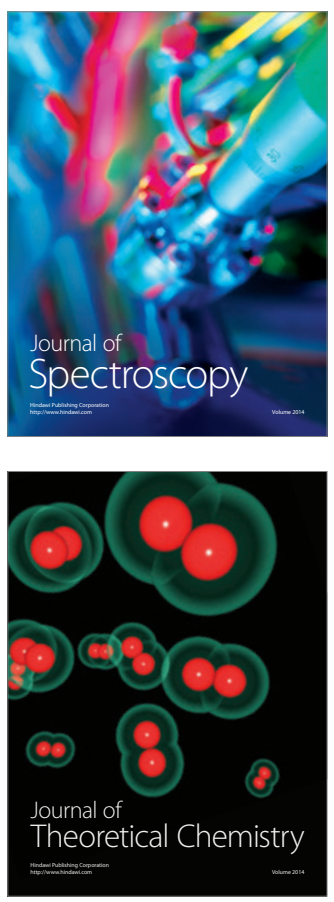
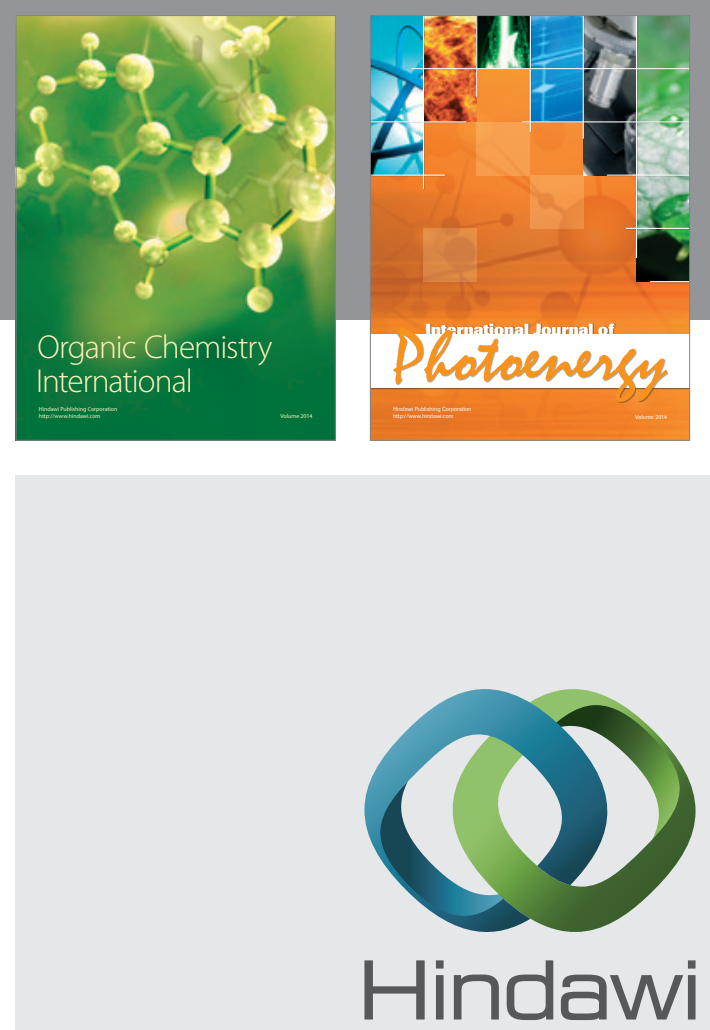

Submit your manuscripts at

http://www.hindawi.com
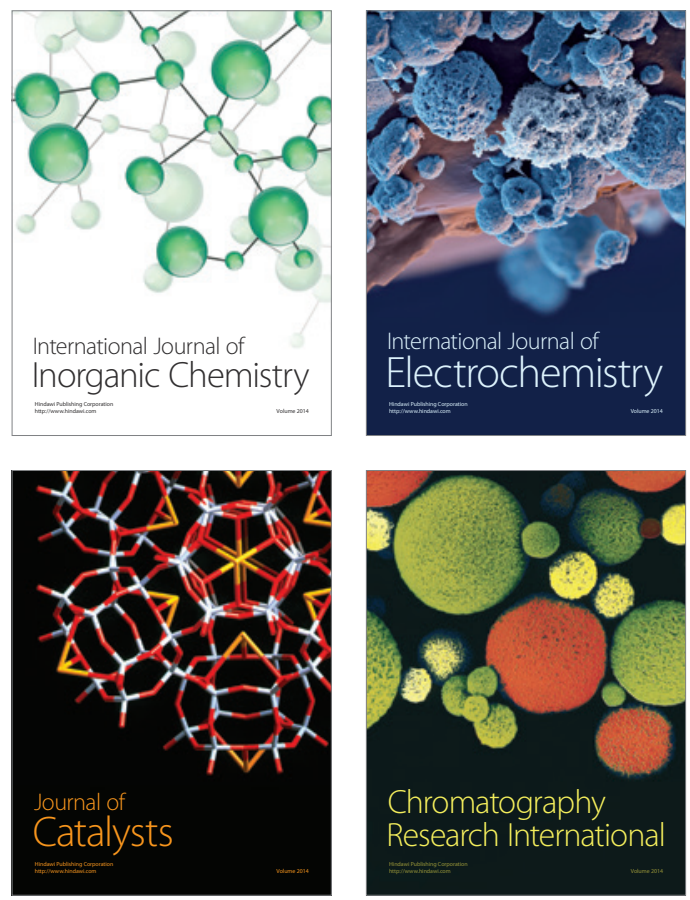
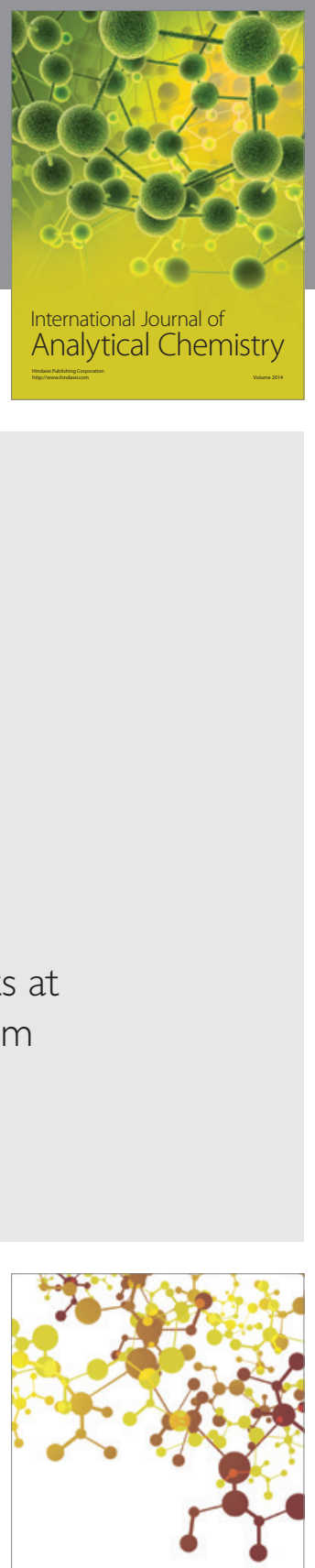

Journal of

Applied Chemistry
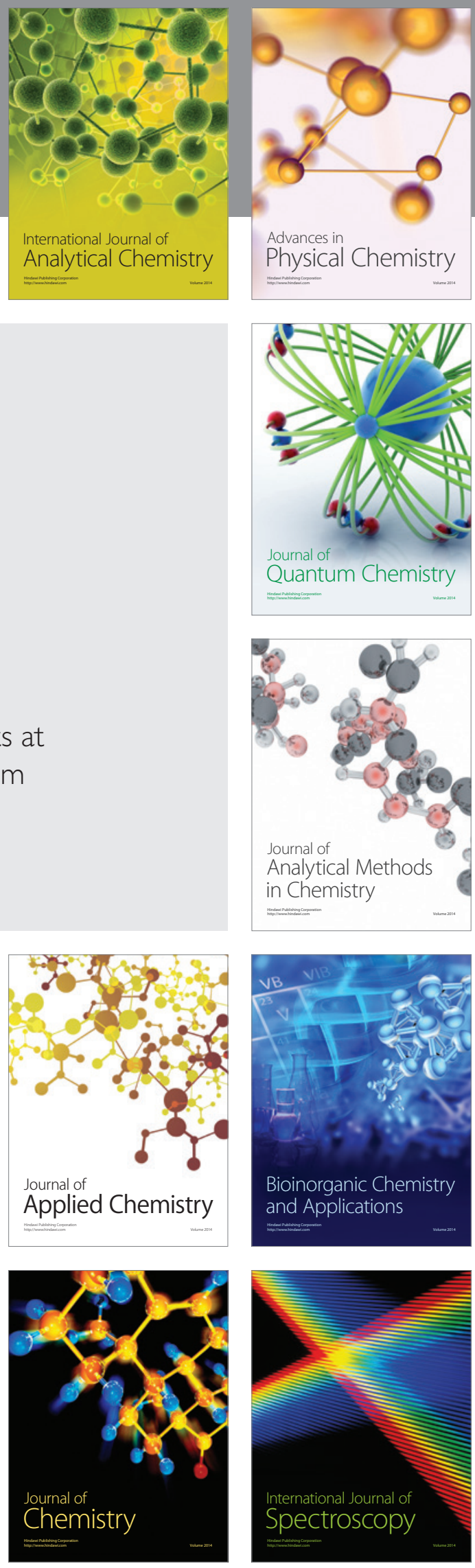